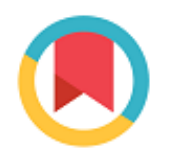

Check for updates

\title{
Comparison of Face to Face vs. Group Training on Self-pulse Rate taking Ability of Patients
}

\author{
Habibollah Saadat ${ }^{1}$, Roxana Sadeghi ${ }^{1,}$, , Maryam Jannatipour \\ ${ }^{1}$, Alireza Abadi ${ }^{2}$, Zahra Saadat ${ }^{1}$, Saeed Alipour Parsa ${ }^{1}$ \\ ${ }^{1}$ Cardiovascular Research Center, Shahid Beheshti University of Medical \\ Sciences, Tehran, Iran \\ 2 Department of Health and Community Medicine, School of Medicine, \\ Shahid Beheshti University of Medical Sciences, Tehran, Iran \\ ${ }^{*}$ Corresponding author: Roxana Sadeghi, MD, Cardiovascular Research Center, \\ Shahid Beheshti University of Medical Sciences, Tehran, Iran. Phone: +98- \\ 2122083106;E-mail: roxan.sadeghi@sbmu.ac.ir
}

DOI: $10.21859 / \mathrm{ijcp}-401$

\begin{tabular}{|c|c|}
\hline Submitted: $12-08-2018$ & \multirow{3}{*}{$\begin{array}{l}\text { Abstract } \\
\text { Introduction: Determining the rate and regularity of peripheral arterial pulses has a } \\
\text { major role in assessing the clinical status of patients with cardiovascular disorders. We }\end{array}$} \\
\hline Accepted: $05-10-2018$ & \\
\hline Keywords: & \\
\hline Pulse Rate & \multirow{2}{*}{$\begin{array}{l}\text { compared two training methods on the ability of patients to take their radial pulse rate } \\
\text { accurately. }\end{array}$} \\
\hline Patient Education as Topic & \\
\hline Cardiovascular Diseases & \multirow{2}{*}{$\begin{array}{l}\text { Methods: Three-hundred patients were randomly divided into two arms. One arm } \\
\text { received individual face-to-face training and the other arm received group training via } \\
\text { displaying an animation movie. Immediately after the training and then after } 48 \text { hours, } \\
\text { the patients were tested by a nurse to find out whether they have learned the correct } \\
\text { technique of taking radial pulse rate or not. }\end{array}$} \\
\hline $\begin{array}{l}\text { (c) 2019. International Journal of } \\
\text { Cardiovascular Practice. }\end{array}$ & \\
\hline & $\begin{array}{l}\text { Results: Immediately after the intervention, } 84.9 \% \text { in face-to-face arm and } 81.8 \% \text { in } \\
\text { group training arm were able to correctly count their radial pulse rate }(\mathrm{P}=0.536) \text {. After } \\
48 \text { hours, } 71.7 \% \text { in face-to-face and } 60.8 \% \text { in group training arm were able to correctly } \\
\text { count their radial pulse rate }(\mathrm{P}=0.051) \text {. }\end{array}$ \\
\hline & $\begin{array}{l}\text { Conclusions: Both methods were effective to improve the ability of the patients to } \\
\text { count their radial pulse rate correctly though face-to-face method was marginally } \\
\text { superior to group training. }\end{array}$ \\
\hline
\end{tabular}

\section{INTRODUCTION}

Determining the rate and regularity of peripheral arterial pulses has a major role in assessing the clinical status of patients with cardiovascular disorders, in particular dysrhythmias. Hence most people cannot detect and count their pulse rhythm correctly, an American Heart Association (AHA) guideline inevitably omitted pulse detecting by ley rescuers in the Cardiopulmonary Resuscitation algorithm [1]. On the other hand self-care is essential to maintenance of health, and prevention and management of chronic diseases. David W. Young who was involved with atrial arrhythmias, referred his personal experience to point the benefits of using selfmeasure of heart rate variability (HRV) to monitor and to manage atrial arrhythmias [2]. However, to correctly control cardiovascular disease (CVD), self-care not only must be done by those who already have CVD but also practiced by all people at all ages [3]. In spite of recent improvements in electronic and instrumental assessment of heart rate, the simple and rather rapid traditional method for assessing pulse rate has remained as a common method amongst clinicians for monitoring heart rate and its abnormalities [4]. The AHA guidelines recommend patients with permanent pace maker to take and record their pulse rate and report it to their healthcare providers [5]. In addition, pulse rate is a simple and practical tool for assessment of heart rate by general population [6]. In 2018, James Cole and et al, showed that opportunistic pulse regulating checks using in clinic templates was associated with a significance increase in detecting atrial fibrillation rhythm [7].

However, the reliability and validity of this method of pulse rate control for assessment of heart rhythm can be affected by duration of pulse measurement as well as observer errors. There are conflicting reports about accuracy of recording the heart rate by counting the pulse rate within 15,30 , and 60 seconds [8]. There is evidence that variability exists in heart rate either in inter-individual or intra-individual pulse rate taking [9, 10]. Several studies indicate the importance of selfmeasured blood pressure in risk stratification of patients [11]. However, there are not enough studies targeting patient education with respect to self-measurement of 
pulse rate and its regularity. We speculated that training sessions held individually or in a group form could enhance the ability of patients in taking their own pulse rate accurately. Hence, the present study aimed to assess the impact of two training methods including face to face training and group training on counting pulse rate and assessment of its regularity in patients with cardiovascular diseases. By studying each method individually and comparing them we will be able to find out whether the implemented trainings are effective or not and also will introduce the better method which can be used by clinicians.

\section{METHODS}

In this prospective interventional study 300 patients who had been referred to two educational hospitals (Shahid Modarres Hospital and Loghman Hakim Hospital) with cardiovascular diseases, were included. On admission, the baseline characteristics including demographic data, socioeconomic status, educational level and the reason for admission were questioned. Initially and using a checklist, patients were questioned and tested for their knowledge and ability to assess radial pulse rate and those who already knew the correct method of taking radial pulse rate were excluded. The patients were randomly divided (using random number table) into two groups. The first group underwent face to face training by a single trained registered nurse with regard to counting radial pulse rate and also determining its regularity. The patients in the second group participated at sessions supervised by a trained staff nurse during which an educational movie using a 3minute animation about taking radial pulse rate and assessing its regularity produced by our research team was displayed for them.

The patients in both groups were practically tested by the same nurse immediately and 48 hours after training (before discharge). In this regard, the difference of 5 pulses or higher between patient and nurse was considered significant. The effects of training methods on quality of taking the radial pulse rate and rhythm assessment were examined and compared between the two training groups and also between the two hospitals. Statistical analysis was performed using the SPSS version 16.0. The student's $t$ - test or Mann-Whitney $U$ test was used to test the differences between quantitative variables. The Chi-square and Fisher's exact test were used for testing the association between categorical variables. To examine the effect of covariates of group of education, gender, hospital location, education, migration to city and age on being able to read pulse correctly, we used logistic regression model. $P$ value less than $<0.05$ was considered as the significant.

\section{RESULTS}

\section{Baseline Information}

In total, 300 patients (200 patients at hospital No. 1 and 100 patients at hospital No. 2) with cardiovascular diseases were consecutively included into the study. The two groups in the two hospitals were comparable in mean age ( $57.37 \pm 0.79$ years versus $49.17 \pm 1.03$ years, $\mathrm{P}=0.926)$, male gender ( $59.5 \%$ versus $55 \%, \mathrm{P}=0.886)$, and secondary to higher educational level ( $53 \%$ versus $64.0 \%, \mathrm{P}=0.229$ ) in hospital No. 1 and hospital N. 2, respectively.

The patients in each group were randomly assigned to train with face to face training method or group training method. Thus, at hospital No. 1, 101 patients (mean age $57.42 \pm 1.10$ years, 60 male) received face to face training and 99 patients $(57.30 \pm 1.15$ years, 59 male $)$ received group training. Also, at hospital No. 2, 51 patients (mean age $48.76 \pm 1.54$ years, 27 male) received face to face training and 49 patients $(49.59 \pm 1.38$ years, 28 male) received group training (Table 1 ).

\section{First Assessment (Immediately after Training)}

Table 2 summarizes the proportions of patients who were able to count their radial pulse rate in the two groups immediately after the interventions. As seen, no significant difference was observed between the two groups.

\section{Second Assessment (48h after Training, before Discharge)}

Table 3 summarizes the proportions of patients who were able to count their radial pulse rate in the two groups 48 hours after the interventions. As seen, no significant difference was observed between the two groups. However, the $\mathrm{P}$ value in face-to-face method was marginal. The significant covariate in the model was education level with odds ratio of 1.91 and $\mathrm{B}=0.652(\mathrm{P}$ $=0.015)$.

Table 1: Baseline Characteristics of Patients in Two Hospitals

\begin{tabular}{|c|c|c|c|}
\hline & Face to face $(\mathrm{N}=152)$ & Group training using a video clip $(\mathrm{N}=148)$ & P Value \\
\hline Gender, male & $57.2 \%$ & $58.8 \%$ & 0.81 \\
\hline Age & $54.5(11.7)$ & $54.7(11.4)$ & 0.86 \\
\hline Educational level & & & 0.72 \\
\hline Lower than junior high school & $42.1 \%$ & $44.6 \%$ & \\
\hline Higher than junior high school & $57.9 \%$ & $55.4 \%$ & \\
\hline
\end{tabular}

Table 2: Comparison of Pulse Rate Recordings by Patients and the Nurse Immediately after Training between the Two Studied Groups

\begin{tabular}{|lccc|}
\hline Intervention group & Face to face $(\mathbf{N}=\mathbf{1 5 2})$ & Group training using a video clip (N = 148) & P value \\
Correct pulse rate recording & $84.9 \%$ & $81.8 \%$ & 0.53 \\
Correct diagnosis of regular rhythm & $98.0 \%$ & $97.9 \%$ & 1.00 \\
\hline
\end{tabular}


Table 3: Comparison of Pulse Rate Recordings by Patients and the Nurse after 48 Hours between the Two Studied Groups

\begin{tabular}{|lccc|}
\hline Intervention group & Face to face $(\mathbf{N}=\mathbf{1 5 2})$ & Group training using a video clip (N=148) & P value \\
Correct pulse rate recording & $71.7 \%$ & $60.8 \%$ & 0.051 \\
Correct diagnosis of regular rhythm & $96.5 \%$ & $96.1 \%$ & 1.00 \\
\hline
\end{tabular}

\section{DISCUSSION}

As previously pointed, different factors may affect the reliability and validity of radial pulse assessment such as time of assessment and also knowledge of observers or patients themselves. According to the potential role of the level of patients' knowledge towards correct assessment of radial pulse rate and its regularity, we goaled to determine the effect of both face to face and group training of patients in two different hospitals on the quality of radial pulse count and validity of radial pulse assessment by the patients.

In assessing the quality of pulse rate counting, our defined cutoff was the difference at least 5 pulses counted by the nurse as the standard and the trained patients. We did not see any statistical difference between the two groups either immediately after completion of the interventions or after 48 hours. Though, the difference after 48 hours was marginal and higher number of patients in face-to-face method was able to take their radial pulse correctly. This superiority may be due to possibility to group educational challenging between training nurse and trained patient or may be due to using film and media and just visual memory.

\section{CONCLUSIONS}

Self- check for pulse rate in patients with established cardiovascular disease may be simple and easy to do. By employing appropriate training programs especially face to face training, the patients' ability to radial pulse counting and assessing its regularity may be successfully achieved and may lead to controlling and preventing adverse cardiovascular events before patients' admission to hospital, but need more trials for assessment that which training methods is better. As pulse rate is an important physical signs, educating patient to achieve the ability of taking their own pulse, preferably via face to face method, can be considered by health care professionals.

\section{Acknowledgment}

Thank you Miss Fatemeh Moebali for participating in self-taking pulse rate patient education program and thank you Mr. Hadi Niktab for producing a clip for using in patient education.

\section{Conflict of Interest}

All authors have no conflict of interest.

H. Saadat; the main conceptual ideas, designed and directed the project, Sadeghi, Z.Saadat, Alipour Parsa; wrote the article, Jannatipour Collected the data, Abadi performed the data analysis.

\section{REFERENCES}

1. Web-based Integrated 2010 \& 2015 American Heart Association Guidelines for Cardiopulmonary Resuscitation and Emergency Cardiovascular Care. Adult Basic Life Support and Cardiopulmonary Resuscitation Quality [Internet]. American Heart Association. 2010 \& 2015 [cited 2018]. Available from: https://eccguidelines.heart.org/index.php/circulation/cprecc-guidelines-2/part-5-adult-basic-life-support-andcardiopulmonary-resuscitation-quality/\#.

2. Young DW. Self-measure of heart rate variability (HRV) and arrhythmia to monitor and to manage atrial arrhythmias: personal experience with high intensity interval exercise (HIIE) for the conversion to sinus rhythm. Front Physiol. 2014;5:251. doi: 10.3389/fphys.2014.00251 pmid: 25071596

3. Riegel B, Moser DK, Buck HG, Dickson VV, Dunbar SB, Lee $\mathrm{CS}$, et al. Self-Care for the Prevention and Management of Cardiovascular Disease and Stroke: A Scientific Statement for Healthcare Professionals From the American Heart Association. J Am Heart Assoc. 2017;6(9). doi: 10.1161/JAHA.117.006997 pmid: 28860232

4. Agelink MW, Malessa R, Baumann B, Majewski T, Akila F, Zeit $\mathrm{T}$, et al. Standardized tests of heart rate variability: normal ranges obtained from 309 healthy humans, and effects of age, gender, and heart rate. Clin Auton Res. 2001;11(2):99-108. pmid: 11570610

5. Living with your pacemaker [Internet]. American Heart Association. 2015 [cited 2018]. Available from: http://www.heart.org/HEARTORG/Conditions/Arrhythmi a/PreventionTreatmentofArrhythmia/Living-With-YourPacemaker_UCM_305290_Article.jsp\#.VklydHmJjIV.

6. The official U.S. government site for Medicare 2018. Available from: https://www.medicare.gov.

7. Cole J, Torabi P, Dostal I, Homer K, Robson J. Opportunistic pulse checks in primary care to improve recognition of atrial fibrillation: a retrospective analysis of electronic patient records. Br J Gen Pract. 2018;68(671):e388-e93. doi: 10.3399/bjgp18X696605 pmid: 29784865

8. Hwu YJ, Coates VE, Lin FY. A study of the effectiveness of different measuring times and counting methods of human radial pulse rates. J Clin Nurs. 2000;9(1):146-52. pmid: 11022503

9. Kobayashi H. Inter- and intra-individual variations of heart rate variability in Japanese males. J Physiol Anthropol. 2007;26(2):173-7. pmid: 17435361

10. Johansson JK, Puukka PJ, Virtanen R, Jula AM. Beat-to-beat, ambulatory hour-to-hour, and home day-to-day variabilities in blood pressure, pulse pressure, and heart rate in comparison with each other and with target-organ damage. Blood Press Monit. 2015;20(3):113-20. doi: 10.1097/MBP.0000000000 000101 pmid: 25943466

11. Asayama K, Thijs L, Brguljan-Hitij J, Niiranen TJ, Hozawa A, Boggia J, et al. Risk stratification by self-measured home blood pressure across categories of conventional blood pressure: a participant-level meta-analysis. PLoS Med. 2014;11(1):e1001591. doi: 10.1371/journal.pmed.1001591 pmid: 24465187 